\title{
Deepak Rajagopal and David Zilberman: Environmental, economic, and policy aspects of biofuels
}

\author{
Now Publishers Inc., Hanover, MA, USA, and Delft, Netherlands, \\ 2008, 115 pp, paperback, ISBN 978-1601981745
}

\author{
Mairon G. Bastos Lima
}

Published online: 31 August 2010

(C) The Author(s) 2010. This article is published with open access at Springerlink.com

The global expansion of biofuel production has spurred a major debate on the implications of this fast and large-scale trend. As an increasing number of countries adopt promotion policies on biofuels and the sector expands rapidly both in volume and geographical distribution, concerns on environmental, social, and economic impacts have surfaced quite strongly, and biofuels have become one of the most controversial issues on the agenda. On the one hand, they promise to reduce fossil fuel dependence and to help mitigate climate change, while potentially providing also benefits in terms of energy security and rural development. On the other hand, "side effects" of large-scale biofuel expansion have given reason for much concern. These include, for instance, land-use changes, additional pressure on freshwater resources, and food security implications from the diversion of food crops into fuel manufacturing. The debate is very complex because there are different types of biofuels, produced under different conditions, and with varied impacts. As such, efforts to shed light on these issues and to analyze the biofuel policies in place are of much relevance.

It is in this context that Rajagopal and Zilberman's book "Environmental, Economic and Policy Aspects of Biofuels" provides a useful review of studies on biofuel and discusses some of the major issues at stake. The authors divide the book into five chaptersthree core chapters on the environmental, economic, and policy aspects of biofuels, preceded by an introduction and followed by a conclusion. They start by giving a general overview of biofuel production, which provides background information on the history of biofuels, a concise review of different feedstocks and conversion technologies, and a brief discussion of existing research on the viability of further biofuel expansion (Chapter 1). This is useful not only to set the larger picture but also for making clear that biofuel impacts vary depending on how they are produced.

Chapter 2 looks at the environmental footprints of those different biofuels, reviewing various life-cycle analyses (LCAs) performed. The authors go through a large number of assessments, particularly on corn-ethanol production in the United States and sugarcane-

\section{G. Bastos Lima $(\bowtie)$}

Institute for Environmental Studies (IVM), Vrije Universiteit Amsterdam,

De Boelelaan 1087, 1081 HV Amsterdam, Netherlands

e-mail: mairon.bastos.lima@ivm.vu.nl 
ethanol from Brazil, and conclude that although biofuels may reduce the carbon-intensity of energy systems, they normally contribute to other environmental problems associated to industrial agriculture, such as soil degradation and ecosystem contamination from agricultural chemical inputs. The authors contend that these other environmental indicators not directly related to greenhouse gas emissions need much more attention. They highlight how LCAs need to be better adjusted to real-world conditions, too, in order to provide more useful assessments; "LCA indicators today are just numbers and not a function of prices and policies" (p. 49). The authors thus give a number of suggestions on how that can be accomplished, and cite examples of how failure to do that may result in inconclusive or misleading LCAs.

Chapter 3, on the economic aspects of biofuel production, is perhaps the most comprehensive chapter in the book-reflecting the authors' main expertise. The chapter surveys various economic studies modeling the impacts of biofuels on agricultural commodity prices, on biofuel competitiveness with oil, and on technology adoption by different actors, among others. The authors examine how these models-well established in agricultural economics research-have been applied to the case of biofuels, and they reveal some shortcomings of this process. Most notably, they call attention to the need to perform more ex-post analyses rather than just simulation-based studies on biofuels.

Chapter 4 is dedicated to the policy aspects of biofuels. Here the authors map out the different policy instruments being used to promote biofuels around the world, including farm policies, trade policies, public R\&D funding, and different carbon and energy policies in place. They evaluate some economic impacts of these policies and analyze the extent to which each of them is effective in meeting various goals related to biofuels (oil use reduction, increase in farm incomes, etc.). For instance, the authors develop the argument that taxes on fossil fuels may be more effective than subsidies on biofuels as a way to mitigate climate change. Although the examples provided are mostly from the United States' context, some of the lessons drawn can be quite useful for policy analysis and policy makers elsewhere.

The authors conclude in their fifth and final chapter that biofuels are diverse and evolving. They coherently summarize the key points from the previous chapters and duly blur the borderlines between environmental, economic, and policy aspects. Their main contribution is to provide useful recommendations for further research on LCAs, for economic studies on biofuels, and to biofuel policy makers and analysts.

The practical recommendations on how to proceed with biofuel expansion, on the other hand, are given somewhat uncritically. For instance, the suggestion that food versus fuel competition may be addressed by increasing agricultural productivity seems to miss the complexity of food security debates on the underlying causes of hunger, poverty etc. Likewise, recommendations to adopt "less restrictive regulation on agricultural biotechnology" (p. v), or the bet placed on next generation biofuels (without examining their implications and risks), are not supported by analysis in the book and could have reflected more the different positions that exist on these issues.

It must be said that, although the authors' ambition is seemingly to provide a thorough review of biofuel issues, they analyze them mainly from the perspective of mainstream economics. If on the one hand that allows the authors to examine LCA studies more critically, through a pragmatic, "real-world" lens, and to provide a useful discussion of economic modeling and economic policies on biofuels, it also creates some shortcomings in terms of the literature they cover, the aspects they discuss, and the "solutions" conceived. For instance, they review a very large number of sources, but mostly quantitative studies. As a consequence, they end up covering basically modeling analyses rather than 
accounts of actual, ongoing impacts of biofuel expansion. Although this may just reflect the literature in the case of economic studies, it is not the case in the environmental impact assessment literature, where ex-post analyses of biofuel production are abundant. This makes the chapter on environmental aspects, focused solely on LCAs and estimates, look somewhat limited. The review and assessment of LCAs is useful, but other dimensions such as the politics and the social aspects of environmental issues are not brought into the discussion. The same can be said of the chapter on policies. It does a good job assessing the economic incentives given by different biofuel policies in place, but the mapping out of "policy aspects" is incomplete without, for instance, the existing regulations on how biofuels are to be produced, such as sustainability requirements being put in place. Finally, partly because the coverage of biofuel issues does not incorporate political and social aspects, the conception of eventual "ways forward" has a hard time leaving the box of technological solutions and conventional, large-scale agriculture-even if the latter is recognized in the book as a cause of many of the environmental impacts.

That said, the book is a valuable effort to condense the difficult and complex issues of biofuels from the perspective of economics. Biofuel life-cycle analysts and quantitative economists will find here helpful analyses and sound recommendations for further research. In particular, the book will please a readership interested in the context of the United States, where most of the examples and discussed studies are from. Policy makers, too, may benefit from this volume, particularly to the extent that economic policy incentives are concerned.

Open Access This article is distributed under the terms of the Creative Commons Attribution Noncommercial License which permits any noncommercial use, distribution, and reproduction in any medium, provided the original author(s) and source are credited. 